\title{
Universality class of quantum criticality for strongly repulsive spin-1 bosons with antiferromagnetic spin-exchange interaction
}

\author{
C. C. N. Kuhn, ${ }^{1,2}$ X. W. Guan, ${ }^{2, *}$ A. Foerster, ${ }^{1}$ and M. T. Batchelor ${ }^{2,3}$ \\ ${ }^{1}$ Instituto de Fisica da UFRGS, Av. Bento Goncalves 9500, Porto Alegre, Rio Grande do Sul, Brazil \\ ${ }^{2}$ Department of Theoretical Physics, Research School of Physics and Engineering, Australian National University, Canberra, \\ Australian Capital Territory 0200, Australia \\ ${ }^{3}$ Mathematical Sciences Institute, Australian National University, Canberra, Australian Capital Territory 0200, Australia
}

(Received 31 January 2012; published 9 April 2012)

\begin{abstract}
Using the thermodynamic Bethe ansatz equations we study the quantum phase diagram, thermodynamics, and criticality of one-dimensional (1D) spin-1 bosons with strongly repulsive density-density and antiferromagnetic spin-exchange interactions. We analytically derive a high-precision equation of state from which the TomonagaLuttinger-liquid physics and quantum critical behavior of the system are computed. We obtain explicit forms for the scaling functions near the critical points yielding the dynamical exponent $z=2$ and correlation length exponent $v=1 / 2$ for the quantum phase transitions driven by either the chemical potential or the magnetic field. Consequently, we further demonstrate that quantum criticality of the system can be mapped out from the finite temperature density and magnetization profiles of the 1D trapped gas. Our results provide the physical origin of quantum criticality in a 1D many-body system beyond the Tomonaga-Luttinger-liquid description.
\end{abstract}

DOI: 10.1103/PhysRevA.85.043606

\section{INTRODUCTION}

The study of spinor Bose gases is an active area of research in the field of cold atoms $[1,2]$. In an optical trap, the laseratom interaction is determined by the induced electric dipole moment, thus the atoms are confined independently of their spin orientations. This has provided exciting opportunities of simulating quantum dynamics of spinor Bose-Einstein condensates in which the "vector" property of spinor atoms can be preserved. Several experimental groups have successfully demonstrated spinor BECs of ${ }^{23} \mathrm{Na}[3,4]$ and ${ }^{87} \mathrm{Rb}[5-7]$ atoms in optical traps. In particular, the exquisite tunability with ultracold atoms confined to low dimensions has provided unprecedented opportunities for testing the theory of one-dimensional (1D) exactly solvable many-body systems [8-17]. These experimental developments have stimulated an extensive study of related exactly solvable models with $\delta$-function interactions, see recent reviews [18,19].

Quantum spinor gases with multispin states exhibit richer quantum effects than their single-component counterparts. Spinor Bose gases with spin-independent short-range interactions have a ferromagnetic ground state, i.e., the ground state is always fully polarized [20,21]. In contrast to the two-component Fermi gases [22], the two-component spinor Bose gas with spin-independent $s$-wave scattering [23-25] has a ferromagnetic ground state as long as the interaction is fully spin independent. However, 1D spinor Bose gases with short-range density-density and spin-exchange interactions $[26,27]$ can display a different ground state, i.e., either a ferromagnetic or an antiferromagnetic ground state solely depending on the spin-exchange interaction. In this context, the spin-1 spinor Bose gas with a short-range $\delta$-function interaction and antiferromagnetic spin-spin interaction is particularly interesting due to the existence of various phases of quantum liquids associated with the Bethe ansatz (BA)

\footnotetext{
*xwe105@physics.anu.edu.au
}

PACS number(s): 03.75.Ss, 03.75.Hh, 02.30.Ik, 34.10.+x

solutions [26,28-30]. At zero temperature, this model exhibits three phases in the chemical potential-magnetic field plane. These are (i) a spin-singlet phase of pairs of bosons with hyperfine states $\left|F=1, m_{F}= \pm 1\right\rangle$ or two $\left|F=1, m_{F}=0\right\rangle$ bosons, (ii) a ferromagnetic phase of fully polarized atoms in the hyperfine state $\left|F=1, m_{F}=1\right\rangle$, and (iii) a mixed phase of spin-singlet pairs and unpaired single atoms, see Fig. 1.

Spinor Bose gases exhibit various phases of strongly correlated quantum liquids and are thus particularly valuable to investigating quantum magnetism and criticality. Near a quantum critical point, the many-body system is expected to show universal scaling behavior in the thermodynamic quantities due to the collective nature of many-body effects. Thus a universal and scale-invariant description of the system is expected through the power-law scaling of thermodynamic properties [31,32]. Most recently, quantum criticality and universal scaling behavior have been experimentally investigated in low-dimensional cold atomic matter [33,34]. These advances build on theoretical schemes for mapping out quantum criticality in cold-atom systems [35-37]. In this framework, exactly solvable models of cold atoms, exhibiting quantum phase transitions, provide a rigorous way to treat quantum criticality in archetypical quantum many-body systems, such as the Gaudin-Yang Fermi gas [38], the Lieb-Liniger Bose gas [39], and a mixture of bosons and fermions [40].

Despite much work on the 1D spin-1 bosons with strongly repulsive density-density and antiferromagnetic spin-exchange interactions [26,28,29], there has been no study of the quantum criticality of the model by using the exact solution. Contrary to the impression one might have, exact solvability does not guarantee that physical quantities of interest can be actually calculated by the BA solutions. The thermodynamic Bethe ansatz (TBA) equations for this model [29] involve an infinite number of coupled nonlinear integral equations that impose a number of challenges to accessing the physics of the model.

In the present paper, building on the method proposed in the study of quantum criticality of the Gaudin-Yang Fermi 


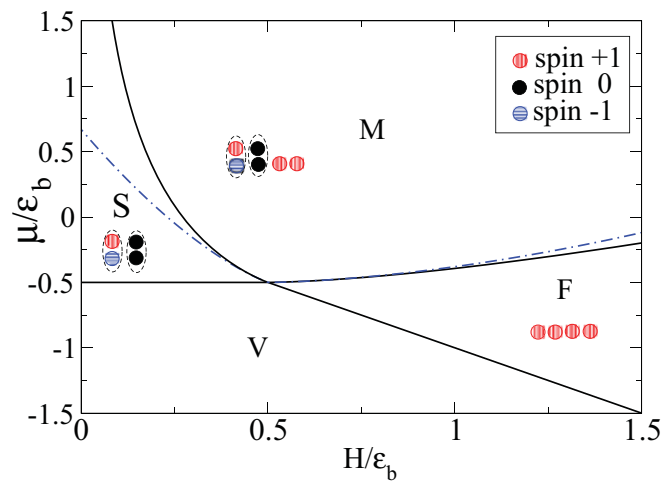

FIG. 1. (Color online) Phase diagram in the $\mu-H$ plane showing the spin-singlet phase $S$ of paired bosons, ferromagnetic phase $F$ of spin-aligned bosons, and a mixed phase $M$ of pairs and unpaired bosons. $V$ stands for the vacuum. The $S-M$ and $F-M$ boundaries are determined by the critical fields in Eqs. (8) and (10), respectively. The $V-S$ and $V-F$ boundaries are given in Eq. (6). The dashed-dotted lines are the extrapolation of the phase boundaries of Eqs. (11) and (12) in the strong coupling regime.

gas [38] and the Lieb-Liniger Bose gas [39], we analytically study the quantum phase diagram, universal thermodynamics, and criticality of spin- 1 bosons with strongly repulsive densitydensity and antiferromagnetic spin-exchange interactions. We derive a high-precision equation of state of the system in experimental accessible conditions, i.e., in the strong coupling regime and low temperatures. We also analytically derive the Tomonaga-Luttinger-liquid (TLL) thermodynamics, quantum critical exponents, and universal scaling functions near the critical points associated with quantum phase transitions driven by the chemical potential and magnetic field. These scaling forms for the thermodynamic properties across the phase boundaries illustrate the physical origin of quantum criticality in this system, where the singular part of the thermodynamic properties involves a sudden change of density of state for either pairs or unpaired single atoms.

The paper is organized as follows. In Sec. II, we present the model and its corresponding TBA equations. In Sec. III, we analytically determine the phase diagram of the model at zero temperature. In Sec. IV, we derive the equation of state and universal TLL thermodynamics in the physical regime, i.e., for strong coupling and low temperatures. In Sec. V, we investigate quantum critical behavior driven by the chemical potential and magnetic field. The scaling functions near the critical points are obtained analytically. Section VI is the conclusion.

\section{MODEL}

We consider $N$ particles of mass $m$ confined in 1D to a length $L$ with $\delta$-interacting type density-density and spinexchange interactions between two atoms. The Hamiltonian is given by $[1,26]$

$\mathcal{H}=-\sum_{i=1}^{N} \frac{\partial^{2}}{\partial x_{i}^{2}}+\sum_{i<j}\left[c_{0}+c_{2} \boldsymbol{S}_{i} \cdot \boldsymbol{S}_{j}\right] \delta\left(x_{i}-x_{j}\right)+E_{z}$, where $\boldsymbol{S}_{i}$ is the spin-1 operator with a $z$ component $(s=1,0$, $-1)$. The interaction parameters are $c_{0}=\left(g_{0}+2 g_{2}\right) / 3$ and $c_{2}=\left(g_{2}-g_{0}\right) / 3$, where $g_{S}=4 \pi \hbar^{2} a_{S} / m$. Here $m$ is the particle mass and $a_{S}$ represents the $s$-wave scattering length in the total spin $S=0,2$ channels. $E_{z}=-H S^{z}$ stands for the Zeeman energy, where $H$ is the external field and $S^{z}$ the total spin in the $z$ component. In the above equation, we have set $\hbar=2 m=1$.

Using the BA hypothesis, Cao et al. [26] solved the model (1) with the antiferromagnetic spin-exchange interaction for $c=c_{0}=c_{2}>0$. The energy eigenspectrum is given in terms of the quasimomenta $\left\{k_{j}\right\}$ of the particles through $E=\sum_{j=1}^{N} k_{j}^{2}$, obeying the following set of coupled BA equations [26]

$$
\begin{aligned}
& \exp \left(\mathrm{i} k_{j} L\right)=\prod_{\ell=1}^{N} \frac{k_{j}-k_{\ell}+4 c^{\prime} \mathrm{i}}{k_{j}-k_{\ell}-4 c^{\prime} \mathrm{i}} \prod_{\alpha=1}^{M} \frac{k_{j}-\Lambda_{\alpha}-2 c^{\prime} \mathrm{i}}{k_{j}-\Lambda_{\alpha}+2 c^{\prime} \mathrm{i}}, \\
& \prod_{\ell=1}^{N} \frac{\Lambda_{\alpha}-k_{\ell}+2 c^{\prime} \mathrm{i}}{\Lambda_{\alpha}-k_{\ell}-2 c^{\prime} \mathrm{i}}=-\prod_{\beta=1}^{M} \frac{\Lambda_{\alpha}-\Lambda_{\beta}+2 c^{\prime} \mathrm{i}}{\Lambda_{\alpha}-\Lambda_{\beta}-2 c^{\prime} \mathrm{i}}
\end{aligned}
$$

Here $c^{\prime}=c / 4, j=1, \ldots, N, \alpha=1 \ldots, M$, and $\left\{\Lambda_{\alpha}\right\}$ are the rapidities for the internal spin degrees of freedom. The quantum number $M$ is a conserved quantity obeying the relation $M=N-S^{z}$. In this model, the antiferromagnetic interaction leads to an effective attraction in the spin-singlet channel so that the singlet bosonic pairs comprise a spin-singlet ground state. In the thermodynamic limit $N, L \rightarrow \infty$, the sets of solutions $\left\{k_{j}\right\}$ and $\left\{\Lambda_{\alpha}\right\}$ of the BA equations (2) take a certain form, where the $k_{j}$ 's and $\Lambda_{\alpha}$ 's can form complex pairs $k_{j}=\lambda_{j} \pm i c^{\prime}$ and $\Lambda_{j}=\lambda_{j} \pm i c^{\prime}$, where $\lambda_{j}$ is real. Notice that each pair of $k_{j}$ 's share the same real part as a corresponding pair of $\Lambda_{j}$ 's. The bound states are associated with a pair of $\left|F=1, m_{F}= \pm 1\right\rangle$ bosons or two $\left|F=1, m_{F}=0\right\rangle$ bosons. In addition to that, we also have real $k_{j}$ 's and $\Lambda$ strings of the form $\Lambda_{\alpha}^{n, j}=\Lambda_{\alpha}^{n}+i(n+1-2 j) c^{\prime}$, $j=1, \ldots, n$ describing spin-wave bound states.

At finite temperatures, the physical states become degenerate. The equilibrium state can be obtained by the condition of minimizing the Gibbs free energy $G=E+E_{z}-\mu N-T S$, where $\mu$ is the chemical potential and $S$ the entropy, see Yang and Yang's grand canonical description [41] of the BA equations for the integrable Bose gas. The Zeeman energy $E_{Z}=-H S_{z}$ and entropy $S$ are given in terms of the densities of charge bound states and spin strings described above which are subject to the BA equations (2). Minimizing the Gibbs free energy leads to a set of coupled nonlinear integral equations, i.e., the TBA equations (see [29] for details)

$$
\begin{aligned}
\varepsilon_{1}(k)= & k^{2}-\mu-H-T a_{4} * \ln \left(1+e^{-\frac{\varepsilon_{1}(k)}{T}}\right) \\
& +T\left[a_{1}-a_{5}\right] * \ln \left(1+e^{-\frac{\varepsilon_{2}(k)}{T}}\right) \\
& -T \sum_{n=1}^{\infty}\left[a_{n-1}+a_{n+1}\right] * \ln \left(1+e^{-\frac{\phi_{n}(k)}{T}}\right), \\
\varepsilon_{2}(k)= & 2\left(k^{2}-c^{\prime 2}-\mu\right)+T\left[a_{1}-a_{5}\right] * \ln \left(1+e^{-\frac{\varepsilon_{1}(k)}{T}}\right) \\
& +T\left[a_{2}-a_{4}-a_{6}\right] * \ln \left(1+e^{-\frac{\varepsilon_{2}(k)}{T}}\right),
\end{aligned}
$$




$$
\begin{aligned}
\phi_{n}(k)= & n+T\left[a_{n-1}+a_{n+1}\right] * \ln \left(1+e^{-\frac{\varepsilon_{1}(k)}{T}}\right) \\
& +T \sum_{n=1}^{\infty} T_{m n} * \ln \left(1+e^{-\frac{\phi_{n}(k)}{T}}\right) .
\end{aligned}
$$

Here $n=1,2, \ldots, \infty$ and the symbol $*$ denotes the convolution $(f * g(x))=\int_{-\infty}^{\infty} f\left(x-x^{\prime}\right) g\left(x^{\prime}\right) d x^{\prime}$, the functions $a_{n}=\frac{1}{\pi} \frac{n\left|c^{\prime}\right|}{\left(n c^{\prime}\right)^{2}+x^{2}}$ and $T_{n m}$ are given in Ref. [29]. These TBA equations are expressed in terms of the dressed energies $\varepsilon_{1}(k)$, $\varepsilon_{2}(k)$, and $\phi_{n}(k)$ for unpaired states, paired states, and spin strings, respectively. They depend on the chemical potential $\mu$, the external field $H$, and spin fluctuations which are ferromagnetically coupled to the unpaired Fermi sea.

The pressure per unit length of the system is given by $p=p_{1}+p_{2}$ with

$$
\begin{aligned}
& p_{1}=\frac{T}{2 \pi} \int_{-\infty}^{\infty} \ln \left(1+e^{-\varepsilon_{1}(k) / T}\right) d k, \\
& p_{2}=\frac{T}{\pi} \int_{-\infty}^{\infty} \ln \left(1+e^{-\varepsilon_{2}(k) / T}\right) d k,
\end{aligned}
$$

corresponding to the pressures for unpaired bosons and spinsinglet pairs, respectively.

\section{PHASE DIAGRAM IN THE $\mu$ - $H$ PLANE}

The ground-state properties and phase diagram at zero temperature can be determined by the dressed energy equations

$$
\begin{aligned}
\varepsilon_{1}(k)= & k^{2}-\mu-H+a_{4} * \varepsilon_{1}(k)+\left[a_{5}-a_{1}\right] * \varepsilon_{2}(k), \\
\varepsilon_{2}(k)= & 2\left(k^{2}-c^{\prime 2}-\mu\right)+\left[a_{5}-a_{1}\right] * \varepsilon_{1}(k) \\
& +\left[a_{6}+a_{4}-a_{2}\right] * \varepsilon_{2}(k),
\end{aligned}
$$

which are obtained from the TBA equations (3) in the limit $T \rightarrow 0$. The negative part of the dressed energies $\varepsilon_{a}(k)$, $a=1,2$ for $k \leqslant Q_{a}$ corresponds to occupied states, while the positive part of $\varepsilon_{a}$ corresponds to unoccupied states. The integration boundaries $Q_{a}$ characterize the "Fermi surfaces" defined by $\varepsilon_{a}\left(Q_{a}\right)=0$. In a canonical ensemble the bosonic pairs form a spin-singlet ground state when the external field is less than a lower critical field. In this phase, the low-energy physics can be characterized by a spin-charge separation theory of the $U(1)$ TLL describing the charge sector and a $O(3)$ nonlinear sigma model describing the spin sector [28]. However, if the external field exceeds an upper critical field, we have solely ferromagnetic single bosons with aligned spins along the external field. For an intermediate magnetic field, the spin-singlet pairs and spin-aligned bosons form a two-component TLL with magnetization [29]. However, in realistic experiments with cold atoms, 1D systems can be realized by tightly confining the atomic cloud in two (radial) dimensions and weakly confining it along the axial direction in a harmonic trap. Therefore, the phase diagram in the $\mu-H$ plane is essential for understanding quantum criticality of the trapped gas at finite temperatures.

We may determine the phase boundaries by analyzing the band fillings in the dressed energy equations (5). The $V-F$ phase boundary is established by the condition $\varepsilon_{1}(k) \leqslant 0$ and $\varepsilon_{2}(k)>0$. Then from Eq. (5) we have $\mu_{c 1}=-H$. The $V-S$ phase boundary is determined by $\varepsilon_{1}(k)>0$ and $\varepsilon_{2}(k) \leqslant 0$, which results in $\mu_{c 2}=-\epsilon_{\mathrm{b}} / 2$, where $\epsilon_{\mathrm{b}}=\hbar^{2} c^{2} /(16 m)$ is the binding energy of the bound pair. For convenience we shall use the dimensionless units in the study of quantum criticality of the system, i.e., $\tilde{\mu} \equiv \mu / \epsilon_{b}$ and $h=H / \epsilon_{\mathrm{b}}$. Thus the critical fields for the phase boundaries $V-F$ and $V$-S read

$$
\tilde{\mu}_{c 1}=-h, \quad \tilde{\mu}_{c 2}=-\frac{1}{2} .
$$

The $F-M$ phase boundary is obtained by the requirement $\varepsilon_{1}\left( \pm Q_{1}\right)=0$ and $\varepsilon_{2}(k) \leqslant 0$, yielding the set of equations

$$
\begin{gathered}
\tilde{\varepsilon}_{1}(x)=8 x^{2}-\tilde{\mu}_{c 3}-h+\frac{1}{\pi} \int_{-\tilde{Q}_{1}}^{\tilde{Q}_{1}} \frac{\tilde{\varepsilon}_{1}\left(x^{\prime}\right)}{1+\left(x-x^{\prime}\right)^{2}} d x^{\prime}, \\
\tilde{Q}_{1}^{2}=\frac{\tilde{\mu}_{c 3}}{8}+\frac{h}{8}-\frac{1}{8 \pi} \int_{-\tilde{Q}_{1}}^{\tilde{Q}_{1}} \frac{\tilde{\varepsilon}_{1}\left(x^{\prime}\right)}{1+\left(\tilde{Q}_{1}-x^{\prime}\right)^{2}} d x^{\prime},
\end{gathered}
$$

which give the critical field for the phase transition from a ferromagnetic phase of spin-aligned bosons into a mixed phase of the pairs and unpaired bosons,

$\tilde{\mu}_{c 3}=-\frac{1}{2}+\frac{2}{5 \pi} \int_{-\tilde{Q}_{1}}^{\tilde{Q}_{1}} \frac{\tilde{\varepsilon}_{1}(x)}{1+16 x^{2} / 25} d x-\frac{2}{\pi} \int_{-\tilde{Q}_{1}}^{\tilde{Q}_{1}} \frac{\tilde{\varepsilon}_{1}(x)}{1+16 x^{2}} d x$,

where $\tilde{Q}_{1}=Q_{1} / c$ and $\tilde{\varepsilon}_{1}(x)$ is given by Eq. (7).

The $S$ - $M$ phase boundary is determined by the conditions $\varepsilon_{1}(k) \leqslant 0$ and $\varepsilon_{2}\left( \pm Q_{2}\right)=0$, from which we obtain the set of equations,

$$
\begin{aligned}
\tilde{\varepsilon}_{2}(x)= & 2\left(8 x^{2}-\frac{1}{2}-\tilde{\mu}_{c 4}\right)+\frac{1}{\pi} \int_{-\tilde{Q}_{2}}^{\tilde{Q}_{2}} \frac{\tilde{\varepsilon}_{2}\left(x^{\prime}\right)}{1+\left(x-x^{\prime}\right)^{2}} d x^{\prime} \\
& +\frac{2}{3 \pi} \int_{-\tilde{Q}_{2}}^{\tilde{Q}_{2}} \frac{\tilde{\varepsilon}_{2}\left(x^{\prime}\right)}{1+4\left(x-x^{\prime}\right)^{2} / 9} d x^{\prime} \\
& -\frac{2}{\pi} \int_{-\tilde{Q}_{2}}^{\tilde{Q}_{2}} \frac{\tilde{\varepsilon}_{2}\left(x^{\prime}\right)}{1+4\left(x-x^{\prime}\right)^{2}} d x^{\prime}, \\
\tilde{Q}_{2}^{2}= & \frac{1}{16}+\frac{\tilde{\mu}_{c 4}}{8}-\frac{1}{24 \pi} \int_{-\tilde{Q}_{2}}^{\tilde{Q}_{2}} \frac{\tilde{\varepsilon}_{2}\left(x^{\prime}\right)}{1+4\left(\tilde{Q}_{2}-x^{\prime}\right)^{2} / 9} d x^{\prime} \\
& -\frac{1}{16 \pi} \int_{-\tilde{Q}_{2}}^{\tilde{Q}_{2}} \frac{\tilde{\varepsilon}_{2}\left(x^{\prime}\right)}{1+\left(\tilde{Q}_{2}-x^{\prime}\right)^{2}} d x^{\prime} \\
& +\frac{1}{8 \pi} \int_{-\tilde{Q}_{2}}^{\tilde{Q}_{2}} \frac{\tilde{\varepsilon}_{2}\left(x^{\prime}\right)}{1+4\left(\tilde{Q}_{2}-x^{\prime}\right)^{2}} d x^{\prime},
\end{aligned}
$$

which provide the critical fields for a phase transition from the spin-singlet phase of paired bosons into a mixed phase of pairs and unpaired bosons,

$\tilde{\mu}_{c 4}=-h+\frac{4}{5 \pi} \int_{-\tilde{Q}_{2}}^{\tilde{Q}_{2}} \frac{\tilde{\varepsilon}_{2}(x)}{1+16 x^{2} / 25} d x-\frac{4}{\pi} \int_{-\tilde{Q}_{2}}^{\tilde{Q}_{2}} \frac{\tilde{\varepsilon}_{2}(x)}{1+16 x^{2}} d x$.

Here $\tilde{Q}_{2}=Q_{2} / c$ and $\tilde{\varepsilon}_{2}(x)$ is given by Eq. (9).

In order to investigate quantum criticality of the system in the strong coupling regime, we need closed form expressions for the critical fields. By Taylor expansion of Eqs. (8) and (10), we obtain the critical field values

$$
\tilde{\mu}_{c 3}=-\frac{1}{2}+\frac{8 \sqrt{2}}{15 \pi}\left(h-\frac{1}{2}\right)^{\frac{3}{2}}+\frac{104}{75 \pi^{2}}\left(h-\frac{1}{2}\right)^{2},
$$




$$
\tilde{\mu}_{c 4}=-h+\frac{32 \sqrt{2}}{15 \pi}\left(\frac{1}{2}-h\right)^{\frac{3}{2}}+\frac{2912}{225 \pi^{2}}\left(\frac{1}{2}-h\right)^{2},
$$

which are in good agreement with the numerical results obtained from Eqs. (8) and (10) in the strong coupling regime. These asymptotic results [Eqs.(11) and (12)] can also be obtained by converting the critical fields obtained in the $H-n$ plane [29] into the $\mu-H$ plane, where the effective chemical potentials $\mu_{1}=\mu+H$ and $\mu_{2}=\mu+\epsilon_{b} / 2$ for unpaired and paired bosons are presented explicitly in Ref. [29].

In the next section we will derive analytical expressions for the equation of state and universal TLL thermodynamics in the physical regime where $t=T / \epsilon_{b} \ll 1$, i.e., for the strong coupling and low-temperature regimes.

\section{EQUATION OF STATE AND TLL THERMODYNAMICS}

The thermodynamics and the high precision of the equation of state of a system provide the key information that can be used to map out quantum critical phenomena and to make comparisons between theory and experiment. Recently, the equation of state of a two-component ultracold Fermi gas has been measured [42,43] using theoretical schemes [44]. Such experimental advances provide exciting opportunities to test universal TLL and quantum critical phenomena in low-dimensional many-body systems.

\section{A. Equation of state}

The lack of analytic solutions of the TBA equations limits the ability to make physical predictions of the model at finite temperatures. In fact, the thermodynamic properties of the model at finite temperature are notoriously difficult to extract due to the presence of the bosonic nature and the spin-spin exchange interaction. Building on the method presented in Ref. [38] and considering the physical region (strong coupling $|c| \gg 1$ and low temperatures), we find that spin fluctuations are strongly suppressed by a strong field, i.e., $H \gg T$. Therefore we can analytically extract the spin-wave bound-state contributions to the unpaired dressed energy, see the third equation in Eqs. (3). Moreover, we notice that the convolution terms converge rapidly once $\varepsilon_{1,2}(k)>0$ in the TBA equations. Therefore, we are allowed to carry out a Taylor expansion with respect to $c$ in the kernel functions $a_{n}$ in the TBA equations at low temperatures. Then, integrating by parts, we may obtain the dressed energies in terms of polylogarithm functions up to order $1 /|c|^{3}$,

$$
\begin{aligned}
\varepsilon_{1}(k) \approx & \frac{\hbar^{2}}{2 m} k^{2}-\mu-H-\frac{2|c| p_{1}}{c^{2}+k^{2}}+\frac{4|c| p_{2}}{c^{2}+16 k^{2}} \\
& -\frac{20|c| p_{2}}{25 c^{2}+16 k^{2}}-\frac{T^{\frac{5}{2}}}{2 \sqrt{\pi}|c|^{3}\left(\frac{\hbar^{2}}{2 m}\right)^{\frac{3}{2}}} \operatorname{Li}_{\frac{5}{2}}\left(-e^{\frac{A_{1}^{0}}{T}}\right) \\
& +\frac{1984 T^{\frac{5}{2}}}{125 \sqrt{2 \pi}|c|^{3}\left(\frac{\hbar^{2}}{2 m}\right)^{\frac{3}{2}}} \operatorname{Li}_{\frac{5}{2}}\left(-e^{\frac{A_{2}^{0}}{T}}\right) \\
& -\mathrm{Te}^{-\frac{H}{T}-\frac{\bar{K}}{4}}\left[\left(1-\frac{2 k^{2}}{c^{2}}\right) I_{0}\left(\frac{\bar{K}}{4}\right)+\frac{2 k^{2}}{c^{2}} I_{1}\left(\frac{\bar{K}}{4}\right)\right],
\end{aligned}
$$

$$
\begin{aligned}
\varepsilon_{2}(k) \approx & \frac{2 \hbar^{2}}{2 m} k^{2}-\frac{\hbar^{2}}{2 m} \frac{c^{2}}{8}-2 \mu+\frac{8|c| p_{1}}{c^{2}+16 k^{2}} \\
& -\frac{40|c| p_{1}}{25 c^{2}+16 k^{2}}+\frac{3968 T^{\frac{5}{2}}}{125 \sqrt{\pi}|c|^{3}\left(\frac{\hbar^{2}}{2 m}\right)^{\frac{3}{2}}} \operatorname{Li}_{\frac{5}{2}}\left(-e^{\frac{A_{1}^{0}}{T}}\right) \\
& +\frac{2|c| p_{2}}{c^{2}+4 k^{2}}-\frac{|c| p_{2}}{c^{2}+k^{2}}-\frac{6|c| p_{2}}{9 c^{2}+4 k^{2}} \\
& +\frac{181 T^{\frac{5}{2}}}{108 \sqrt{2 \pi}|c|^{3}\left(\frac{\hbar^{2}}{2 m}\right)^{\frac{3}{2}}} \operatorname{Li}_{\frac{5}{2}}\left(-e^{\frac{A_{2}^{0}}{T}}\right) .
\end{aligned}
$$

Here $\operatorname{Li}_{s}(z)=\sum_{k=1}^{\infty} z^{k} / k^{s}$ is the polylogarithm function. The terms $\bar{K}=8 p_{1} /(T|c|)$ and $I_{n}(z)=\sum_{\gamma=0}^{\infty} \frac{(z / 2)^{n+2 \gamma}}{\gamma !(n+\gamma) !}$ are obtained from the so-called "string" or spin-wave contributions.

Using the above dressed energies and integrating by parts, we may calculate the pressure (4) in a straightforward way, with the result

$$
\begin{gathered}
p_{1} \approx-\frac{T^{\frac{3}{2}} f_{\frac{3}{2}}^{1}}{\left(\frac{4 \pi \hbar^{2}}{2 m}\right)^{\frac{1}{2}}}\left[1-\frac{p_{1}}{|c|^{3}} \frac{2 m}{\hbar^{2}}+\frac{3968 p_{2}}{125|c|^{3}} \frac{2 m}{\hbar^{2}}\right], \\
p_{2} \approx-\frac{T^{\frac{3}{2}} f_{\frac{3}{2}}^{2}}{\left(\frac{2 \pi \hbar^{2}}{2 m}\right)^{\frac{1}{2}}}\left[1+\frac{3968 p_{1}}{125|c|^{3}} \frac{2 m}{\hbar^{2}}+\frac{181 p_{2}}{108|c|^{3}} \frac{2 m}{\hbar^{2}}\right],
\end{gathered}
$$

where we have denoted $f_{s}^{i}=\operatorname{Li}_{s}\left(-e^{\frac{A_{i}}{T}}\right)$ with $i=1,2$ and

$$
\begin{aligned}
A_{1}= & \mu+H+\frac{2 p_{1}}{|c|}-\frac{16 p_{2}}{5|c|}+\mathrm{Te}^{-\frac{H}{T}} e^{-\frac{\bar{K}}{4}} I_{0}\left(\frac{\bar{K}}{4}\right) \\
& +\frac{T^{\frac{5}{2}}}{|c|^{3}\left(\frac{\hbar^{2}}{2 m}\right)^{\frac{3}{2}}}\left[\frac{1}{2 \sqrt{\pi}} f_{\frac{5}{2}}^{1}-\frac{1984}{125 \sqrt{2 \pi}} f_{\frac{5}{2}}^{2}\right], \\
A_{2}= & \frac{\hbar^{2}}{2 m} \frac{c^{2}}{8}+2 \mu-\frac{32 p_{1}}{5|c|}-\frac{p_{2}}{3|c|} \\
& -\frac{T^{\frac{5}{2}}}{|c|^{3}\left(\frac{\hbar^{2}}{2 m}\right)^{\frac{3}{2}}}\left[\frac{3968}{125 \sqrt{\pi}} f_{\frac{5}{2}}^{1}+\frac{181}{108 \sqrt{2 \pi}} f_{\frac{5}{2}}^{2}\right] .
\end{aligned}
$$

Thus an infinite number of TBA equations have been simplified to two coupled equations, making the thermodynamics of the model analytically accessible.

The above expressions for the pressures in Eqs. (15) and (16) provide the precise equation of state of the system from which universal TLL thermodynamics and scaling functions near critical points can be further derived analytically. We present a high-precision equation of state in the later discussions of the singularities of thermodynamic properties near the quantum critical point as the temperature tends to zero. To evaluate physical properties we substitute Eqs. (17) and (18) into Eqs. (15) and (16). This provides two coupled equations for $p_{1}$ and $p_{2}$, which can be solved by iteration. We discuss the quantum criticality of the system using these pressures in the next section.

\section{B. Universal TLL thermodynamics}

In general, the free energy of a 1D many-body system at low temperatures can be naturally attributed to low-lying excitations near the Fermi points. From calculations of the finite temperature corrections to the free energy, one can extract 
universal TLL thermodynamics. This low-energy physics can also be obtained from conformal field theory [45]. Here we further develop an efficient way to obtain the universal TLL thermodynamics in the mixed phase of the pairs and unpaired bosons from the TBA equations (3). For temperatures $T \ll 1$ and the strong coupling regime $|c| \gg 1$, the gapless phase is in the region $H \sim c^{2} \gg T$. Therefore, we can ignore the spin-wave bound-state contributions in this phase. The first few terms coming from an asymptotic expansion in the TBA equations (3) are given in terms of $1 /|c|$ corrections by

$$
\begin{gathered}
\varepsilon_{1}(k) \approx \frac{\hbar^{2}}{2 m} k^{2}-\mu-H-\frac{2 p_{1}}{|c|}+\frac{16 p_{2}}{5|c|}, \\
\varepsilon_{2}(k) \approx \frac{2 \hbar^{2}}{2 m} k^{2}-\frac{\hbar^{2}}{2 m} \frac{c^{2}}{8}-2 \mu+\frac{32 p_{1}}{5|c|}+\frac{p_{2}}{3|c|} .
\end{gathered}
$$

Higher order corrections can be calculated in a straightforward manner [see the analysis in Eqs. (15) and (16)]. However, they are not necessary in the present discussion. From Eq. (4) we obtain the pressures for spin-aligned single bosons and spin-singlet pairs

where

$$
\begin{aligned}
& p_{1}=\left(\frac{\hbar^{2}}{2 m}\right)^{-1 / 2} \frac{1}{\pi} \int_{0}^{\infty} \frac{\sqrt{\varepsilon_{1}^{0}} d \varepsilon_{1}^{0}}{1+e^{\left(\varepsilon_{1}^{0}-A_{1}^{0}\right) / T}}, \\
& p_{2}=\left(\frac{\hbar^{2}}{2 m}\right)^{-1 / 2} \frac{\sqrt{2}}{\pi} \int_{0}^{\infty} \frac{\sqrt{\varepsilon_{2}^{0}} d \varepsilon_{2}^{0}}{1+e^{\left(\varepsilon_{2}^{0}-A_{2}^{0}\right) / T}},
\end{aligned}
$$

$$
\begin{gathered}
A_{1}^{0} \approx \mu+H+\frac{2 p_{1}}{|c|}-\frac{16 p_{2}}{5|c|}, \\
A_{2}^{0} \approx 2 \mu+\frac{\hbar^{2}}{2 m} \frac{c^{2}}{8}-\frac{32 p_{1}}{5|c|}-\frac{p_{2}}{3|c|} .
\end{gathered}
$$

The integrals in Eqs. (21) and (22) can be calculated explicitly via the Sommerfeld expansion. We assume that there are two "Fermi seas," i.e., a Fermi sea of bound pairs with an effective chemical potential $A_{2}^{0}$ and a Fermi sea of unpaired bosons with an effective chemical potential $A_{1}^{0}$. From Eqs. (19)-(24) and the relations $n_{1}=\frac{\partial p}{\partial H}$ and $n=n_{1}+2 n_{2}=$ $\frac{\partial p}{\partial \mu}$, by a cumbersome iteration we can obtain closed forms for the pressures

$$
\begin{aligned}
p_{1}= & \frac{2 \pi^{2} n_{1}^{3}}{3}\left(1-\frac{6 n_{1}}{|c|}+\frac{96 n_{2}}{5|c|}\right) \\
& +\frac{T^{2}}{6 n_{1}}\left(1+\frac{2 n_{1}}{|c|}-\frac{32 n_{2}}{5|c|}\right), \\
p_{2}= & \frac{\pi^{2} n_{2}^{3}}{3}\left(1+\frac{48 n_{1}}{5|c|}+\frac{n_{2}}{|c|}\right) \\
& +\frac{T^{2}}{3 n_{2}}\left(1-\frac{16 n_{1}}{5|c|}-\frac{n_{2}}{3|c|}\right) .
\end{aligned}
$$

The Helmholtz free energy per unit length is given by $f=$ $n \mu-p$. After a lengthy iteration, we obtain a universal leading temperature correction to the free energy of the form

$$
f=f_{0}-\frac{\pi T^{2}}{6}\left(\frac{1}{v_{1}}+\frac{1}{v_{2}}\right)
$$

with $f_{0}$ the ground state already obtained in Ref. [29]. Here

$$
v_{1}=2 \pi n_{1}\left(1+\frac{2\left(32 n_{2}-10 n_{1}\right)}{5|c|}\right)
$$

and

$$
v_{2}=\pi n_{2}\left(1+\frac{2\left(48 n_{1}+5 n_{2}\right)}{15|c|}\right)
$$

are the charge velocities for unpaired and paired bosons. The entropy per unit length is given by $s=-\frac{\partial f}{\partial T}$ where

$$
s=\frac{\pi T}{3}\left(\frac{1}{v_{1}}+\frac{1}{v_{2}}\right) .
$$

We observe that in this gapless phase, spin-wave boundstate fluctuations are suppressed due to a strong external field. The suppression of spin fluctuations leads to a universality class of a two-component TLL in the mixed phase of pairs and unpaired bosons, which we denote by $\mathrm{TLL}_{M}$. At low temperatures, the spin-singlet phase persists as a single component TLL (denoted by $\mathrm{TLL}_{S}$ ) below a crossover temperature. The fully polarized single atoms can persist in a TLL phase (denoted by $\mathrm{TLL}_{F}$ ) below another crossover temperature. However, the TLL is not appropriate for describing quantum criticality, since it does not include proper thermal fluctuations for the quantum critical regime. In general the TLL persists below the crossover temperature at which the relation of the linear temperature-dependent entropy (or specific heat) breaks down.

In Fig. 2 we present the entropy as a function of the temperature using these two different approaches: the polylogarithm function result [Eqs. (15)-(18)] and the Sommerfeld expansion (30). The crossover temperature $t^{*}$ determines the boundaries between the TLL regime and the quantum critical regime; see the contour plots of entropy in the $t-\mu$ plane for two different values of the external field in Fig. 3. The crossover boundaries are established by the points at which the TLL entropy (30) breaks down, i.e., the entropy is no longer linear temperature dependent. At finite temperatures, the system exhibits the characteristic $\mathrm{V}$-shaped behavior of quantum criticality.

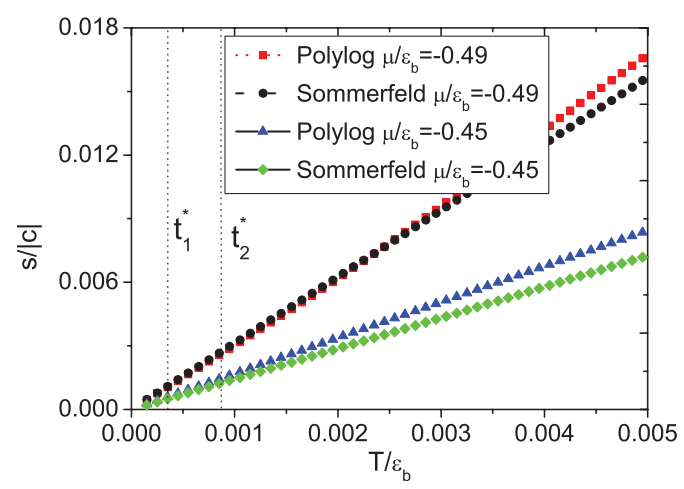

FIG. 2. (Color online) Entropy vs temperature from TLL entropy [Eq. (30)] and entropy calculated from the equation of state [Eqs. (15)-(18)] for $\mu=-0.49$ and $\mu=-0.45$. The universal linear temperature-dependent TLL entropy is broken down at the crossover temperatures $t^{*}$ which separate the TLL phase and the quantum critical regime, see Fig. 3. 


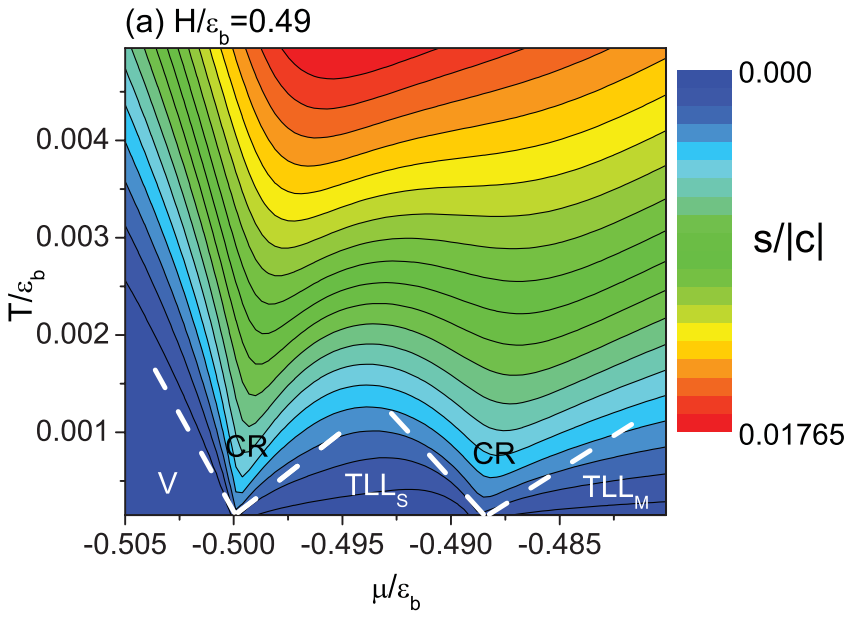

(b) $\mathrm{H} / \varepsilon_{\mathrm{b}}=0.51$

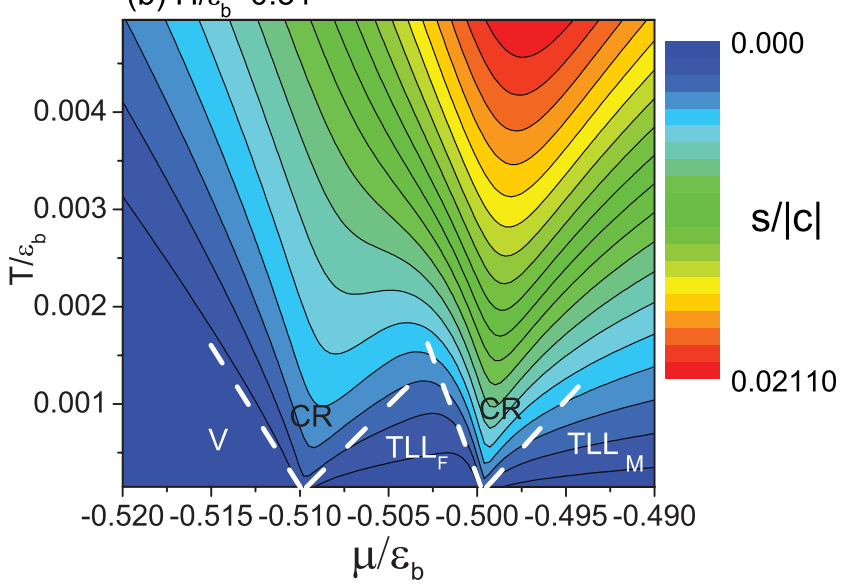

FIG. 3. (Color online) Contour plot of entropy $S$ in the $t$ - $\mu$ plane from the equations of state (15) and (16) for two values of the external magnetic field (a) $h=0.49$ and (b) $h=0.51$. The dashed lines are the crossover temperatures determined in Fig. 2. The intersection points between two dashed lines are the critical points in the $\mu-H$ plane, see Fig. 1. (a) The dashed line separates the $\mathrm{TLL}_{S}$ and $\mathrm{TLL}_{M}$ from the quantum critical regimes. (b) The dashed line separates the TLL $F$ and $\mathrm{TLL}_{M}$ from the quantum critical regimes. The left-most-dashed line in (a) and (b) separates the vacuum $\mathrm{V}$ from the $\mathrm{TLL}_{S}$ and $\mathrm{TLL}_{F}$, respectively.

\section{QUANTUM CRITICALITY}

Quantum criticality describes the critical behavior near a quantum phase transition, i.e., it describes collective behavior of a large number of interacting particles at temperatures sufficiently low, such that quantum mechanics plays a crucial role in determining the distinguishing characteristics [31]. The quantum phase transition occurs at absolute zero temperature as the parameters of the system are varied. In the critical regime, a universal and scale-invariant description of the system is expected through the power-law scaling of thermodynamical properties. From the phase diagram of spin-1 bosons, see Fig. 1, we observe that the quantum phase transition occurs as the driving parameters $\tilde{\mu}$ and $\tilde{h}$ cross the phase boundaries at zero temperature. Although there is no true finite temperature quantum phase transition in a 1D model, quantum criticality of a $1 \mathrm{D}$ many-body system is associated with a universal crossover $T^{*}$ that separates the excitation spectrum from relativistic to nonrelativistic dispersion $[46,47]$. In the present spin-1 model of bosons, we can interpret the spin-singlet phase as a Tonks-Girardeau (TG) gas of hard-core bosons with mass $2 m$ and the spin-aligned ferromagnetic phase as a TG gas of single atoms with mass $m$. The mixed phase of two coupled TG gases is made of particles with mass $m$ and $2 m$.

\section{A. Criticality driven by a chemical potential}

Quantum critical behavior is uniquely characterized by the critical exponents depending only on the dimensionality and the symmetry of the excitation spectrum. This is reflected by singularities in the thermodynamic quantities, such as density $n$, compressibility $\kappa=\partial n / \partial \mu$, and magnetization $M$. They can be obtained from the derivatives of the pressure $p$ with respect to $\mu$ and $H$. In order to identify universal scaling of the thermodynamic properties in the quantum critical regime, we will only take into account the first few terms in the equations of state (15) and (16). To this end, the total pressure is simplified as

$$
\tilde{p}=-\frac{t^{\frac{3}{2}}}{2 \sqrt{2 \pi}}\left(\frac{1}{2} f_{\frac{3}{2}}^{1}+\frac{1}{\sqrt{2}} f_{\frac{3}{2}}^{2}\right),
$$

where we have defined a dimensionless pressure $\tilde{p}=p /|c| \epsilon_{b}$ with the potentials

$$
\begin{aligned}
& \tilde{A}_{1}=\tilde{\mu}+h+2 \tilde{p}_{1}-\frac{16 \tilde{p}_{2}}{5}, \\
& \tilde{A}_{2}=1+2 \tilde{\mu}-\frac{32 \tilde{p}_{1}}{5}-\frac{\tilde{p}_{2}}{3},
\end{aligned}
$$

and we have denoted the function $f_{s}^{i}=\operatorname{Li}_{s}\left(-e^{\frac{A_{i}}{T}}\right)$.

By iterating equations (31)-(33), we obtain the dimensionless density $\tilde{n} \equiv n /|c|$, where

$$
\tilde{n}=-\frac{\sqrt{t}}{2 \sqrt{2 \pi}}\left(\frac{1}{2} f_{1 / 2}^{1} \Delta_{1}+\sqrt{2} f_{1 / 2}^{2} \Delta_{2}\right),
$$

with

$$
\begin{aligned}
\Delta_{1}= & 1-\frac{t^{1 / 2}}{2 \sqrt{2 \pi}} f_{1 / 2}^{1}+\frac{t}{8 \pi}\left(f_{1 / 2}^{1}\right)^{2}+\frac{8 t^{1 / 2}}{5 \sqrt{\pi}} f_{1 / 2}^{2} \\
& +\frac{2 t}{15 \pi}\left(f_{1 / 2}^{2}\right)^{2}+\frac{12 t}{25 \pi \sqrt{2}} f_{1 / 2}^{1} f_{1 / 2}^{2}, \\
\Delta_{2}= & +\frac{4 t^{1 / 2}}{5 \sqrt{2 \pi}} f_{1 / 2}^{1}-\frac{t}{5 \pi}\left(f_{1 / 2}^{1}\right)^{2}+\frac{t^{1 / 2}}{12 \sqrt{\pi}} f_{1 / 2}^{2} \\
& +\frac{t}{144 \pi}\left(f_{1 / 2}^{2}\right)^{2}+\frac{101 t}{75 \pi \sqrt{2}} f_{1 / 2}^{1} f_{1 / 2}^{2} .
\end{aligned}
$$

The total density (34) depends on the density of single atoms and the density of paired atoms. Furthermore, using the standard thermodynamic relations, we obtain the magnetization $\tilde{M} \equiv M /|c|$,

$$
\begin{aligned}
\tilde{M}= & -\frac{\sqrt{t}}{2 \sqrt{2 \pi}}\left\{\frac { 1 } { 2 } f _ { 1 / 2 } ^ { 1 } \left[1-\frac{t^{1 / 2}}{2 \sqrt{2 \pi}} f_{1 / 2}^{1}+\frac{t}{8 \pi}\left(f_{1 / 2}^{1}\right)^{2}\right.\right. \\
& \left.+\frac{32 t}{25 \pi \sqrt{2}} f_{1 / 2}^{1} f_{1 / 2}^{2}\right]+\sqrt{2} f_{1 / 2}^{2}\left[\frac{4 t^{1 / 2}}{5 \sqrt{2 \pi}} f_{1 / 2}^{1}\right. \\
& \left.\left.-\frac{t}{5 \pi}\left(f_{1 / 2}^{1}\right)^{2}+\frac{t}{15 \pi \sqrt{2}} f_{1 / 2}^{1} f_{1 / 2}^{2}\right]\right\},
\end{aligned}
$$


and the susceptibility $\tilde{\chi} \equiv \chi \epsilon_{b} /|c|$,

$$
\begin{aligned}
\tilde{\chi}= & -\frac{1}{2 \sqrt{2 \pi t}}\left\{\frac{1}{2} f_{-1 / 2}^{1}\left[1-\frac{3 t^{1 / 2}}{2 \sqrt{2 \pi}} f_{1 / 2}^{1}+\frac{3 t}{4 \pi}\left(f_{1 / 2}^{1}\right)^{2}+\frac{8 t^{1 / 2}}{5 \sqrt{\pi}} f_{1 / 2}^{2}+\frac{2 t}{15 \pi}\left(f_{1 / 2}^{1}\right)^{2}+\frac{36 t}{25 \pi \sqrt{2}} f_{1 / 2}^{1} f_{1 / 2}^{2}\right]\right. \\
& \left.+f_{-1 / 2}^{2} \frac{32 t}{25 \pi \sqrt{2}}\left(f_{1 / 2}^{1}\right)^{2}\right\} .
\end{aligned}
$$

By a lengthy calculation, the compressibility $\tilde{\kappa} \equiv \kappa \epsilon_{b} /|c|$ is given by

$$
\begin{aligned}
\tilde{\kappa}= & -\frac{1}{2 \sqrt{2 \pi t}}\left\{\frac{1}{2} f_{-1 / 2}^{1}\left[\left(\Delta_{1}\right)^{2}-\frac{t^{1 / 2}}{2 \sqrt{2 \pi}} f_{1 / 2}^{1}\left(1-\frac{3 t^{1 / 2}}{2 \sqrt{2 \pi}} f_{1 / 2}^{1}+\frac{16 t^{1 / 2}}{25 \sqrt{\pi}} f_{1 / 2}^{2}\right)+\frac{2 t^{1 / 2}}{5 \sqrt{\pi}} f_{1 / 2}^{2}\left(1-\frac{3 t^{1 / 2}}{2 \sqrt{2 \pi}} f_{1 / 2}^{1}+\frac{197 t^{1 / 2}}{60 \sqrt{\pi}} f_{1 / 2}^{2}\right)\right]\right. \\
& \left.+2 \sqrt{2} f_{-1 / 2}^{2}\left[\left(\Delta_{2}\right)^{2}+\frac{4 t^{1 / 2}}{5 \sqrt{2 \pi}} f_{1 / 2}^{1}\left(1+\frac{11 t^{1 / 2}}{10 \sqrt{2 \pi}} f_{1 / 2}^{1}+\frac{t^{1 / 2}}{4 \sqrt{\pi}} f_{1 / 2}^{2}\right)+\frac{t^{1 / 2}}{12 \sqrt{\pi}} f_{1 / 2}^{2}\left(1+\frac{424 t^{1 / 2}}{25 \sqrt{2 \pi}} f_{1 / 2}^{1}+\frac{t^{1 / 2}}{4 \sqrt{\pi}} f_{1 / 2}^{2}\right)\right]\right\} .
\end{aligned}
$$

These thermodynamic properties pave the way to extracting universal scaling functions in the vicinity of the critical points $\tilde{\mu}_{c}$. Following the procedure discussed in Ref. [38], quantum criticality of these thermodynamic quantities can be obtained in the limit $T \rightarrow 0$ and $T>\left|\tilde{\mu}-\tilde{\mu}_{c}\right|$ across each of the phase boundaries, with the following results:

$$
\begin{gathered}
V-F:\left\{\begin{array}{l}
\tilde{n} \simeq-\frac{\sqrt{t}}{4 \sqrt{2 \pi}} \operatorname{Li}_{\frac{1}{2}}\left(-e^{\frac{\tilde{\tilde{u}} \tilde{\mu}_{c 1}}{t}}\right), \\
\tilde{M} \simeq-\frac{\sqrt{t}}{4 \sqrt{2 \pi}} \operatorname{Li}_{\frac{1}{2}}\left(-e^{\frac{\tilde{\mu}-\tilde{\mu}_{c 1}}{t}}\right),
\end{array}\right. \\
V-S:\left\{\begin{array}{l}
\tilde{n} \simeq-\frac{\sqrt{t}}{2 \sqrt{t}} \operatorname{Li}_{\frac{1}{2}}\left(-e^{\frac{2\left(\tilde{\mu}-\tilde{\mu}_{c 2}\right)}{t}}\right), \\
\tilde{M} \simeq \frac{2 t}{5 \pi \sqrt{2}} \operatorname{Li}_{\frac{1}{2}}\left(-e^{\frac{2\left(\tilde{\mu}-\tilde{\mu}_{c 2}\right)}{t}}\right) \sim 0,
\end{array}\right. \\
F-M:\left\{\begin{array}{l}
\tilde{n} \simeq \tilde{n}_{03}-\lambda_{1} \sqrt{t} \operatorname{Li}_{\frac{1}{2}}\left(-e^{\frac{2\left(\tilde{\mu}-\tilde{\mu}_{c 3)}\right.}{t}}\right), \\
\tilde{M} \simeq \tilde{M}_{03}+\lambda_{2} \sqrt{t} \operatorname{Li}_{\frac{1}{2}}\left(-e^{\frac{2\left(\tilde{\mu}-\tilde{\mu}_{c 3}\right)}{t}}\right),
\end{array}\right. \\
S-M:\left\{\begin{array}{l}
\tilde{n} \simeq \tilde{n}_{04}-\lambda_{3} \sqrt{t} \operatorname{Li}_{\frac{1}{2}}\left(-e^{\frac{\tilde{\mu}-\tilde{\mu}_{c 4}}{t}}\right), \\
\tilde{M} \simeq-\lambda_{4} \sqrt{t} \operatorname{Li}_{\frac{1}{2}}\left(-e^{\frac{\tilde{\mu}-\tilde{\mu}_{c 4}}{t}}\right) .
\end{array}\right.
\end{gathered}
$$

Here $\tilde{M}_{03}=\tilde{n}_{03}, \tilde{n}_{04}, \lambda_{i}$ (with $\left.i=1, \ldots, 4\right), a$, and $b$ are constants, independent of $\tilde{\mu}$ and $t$. They are given explicitly by

$$
\begin{aligned}
& \tilde{n}_{03}=\frac{\sqrt{a}}{2 \pi \sqrt{2}}\left(1+\frac{\sqrt{a}}{\pi \sqrt{2}}+\frac{a}{2 \pi^{2}}\right), \\
& \tilde{n}_{04}=\frac{\sqrt{b}}{\pi}\left(1-\frac{\sqrt{b}}{6 \pi}+\frac{b}{36 \pi^{2}}\right), \\
& \lambda_{1}=\frac{1}{2 \sqrt{\pi}}\left(1-\frac{16 \sqrt{a}}{5 \pi \sqrt{2}}-\frac{8 a}{25 \pi^{2}}\right), \\
& \lambda_{2}=\frac{4 \sqrt{a}}{5 \sqrt{2} \pi^{3 / 2}}\left(1-\frac{3 \sqrt{a}}{5 \pi \sqrt{2}}\right), \\
& \lambda_{3}=\frac{1}{4 \sqrt{2 \pi}}\left(1-\frac{32 \sqrt{b}}{5 \pi}+\frac{848 b}{75 \pi^{2}}\right), \\
& \lambda_{4}=\frac{1}{4 \sqrt{2 \pi}}\left(1-\frac{16 \sqrt{b}}{5 \pi}+\frac{8 b}{15 \pi^{2}}\right),
\end{aligned}
$$

with

$$
\begin{aligned}
& a=\left(h-\frac{1}{2}\right)\left(1+\frac{13 \sqrt{2}}{15 \pi} \sqrt{h-\frac{1}{2}}\right), \\
& b=2\left(\frac{1}{2}-h\right)\left(1+\frac{91 \sqrt{2}}{45 \pi} \sqrt{\frac{1}{2}-h}\right) .
\end{aligned}
$$

In the above equations $\tilde{n}_{03}$ and $\tilde{n}_{04}$ are the background densities near the critical points $\mu_{3}$ and $\mu_{4}$, respectively. At quantum criticality, the above densities can be cast into a universal scaling form (see $[31,32,36])$, e.g.,

$$
n(\mu, T)=n_{0}+T^{\frac{d}{z}+1-\frac{1}{v z}} \mathcal{G}\left(\frac{\mu-\mu_{c}}{T^{\frac{1}{v z}}}\right) .
$$

Here the dimensionality $d=1$ and the scaling function $\mathcal{G}(x)=$ $\lambda \operatorname{Li}_{\frac{1}{2}}(x)$ with a constant $\lambda$. Consequently the dynamical critical exponent $z=2$ and the correlation length exponent $v=1 / 2$ can be read off from the universal scaling form (43). We observe that the spin- 1 Bose gas belongs to the same universality class as spin-1/2 attractive fermions [38] due to the hard-core nature of the two coupled Tonks-Girardeau gases.

In Figs. 4 and 5 we plot the "scaled density" $T^{-\left(\frac{d}{z}+1-\frac{1}{v z}\right)}\left[n(\mu, T)-n_{0}\right]$ versus $\tilde{\mu}$ for different values of the temperature near the critical points $\tilde{\mu}_{c 1}, \tilde{\mu}_{c 2}, \tilde{\mu}_{c 3}$, and $\tilde{\mu}_{c 4}$. We observe that after an appropriate subtraction of the background density all curves at different temperatures intersect at the critical points, which is the hallmark of criticality. In the regime of low polarization, i.e., $P<P_{c}$, the true phase transitions from the vacuum into the spin-singlet paired phase and from the pure paired phase into the mixture of spin-singlet pairs and spin-aligned bosons occur as the chemical potential passes the lower critical point $\tilde{\mu}_{c 2}$ [Eq. (6)] and the upper critical point $\tilde{\mu}_{c 4}$ [Eq. (12)], respectively, see Fig. 4.

For large polarization, i.e., $P>P_{c}$, the phase transitions from vacuum into the ferromagnetic spin-aligned boson phase and from the spin-aligned boson phase into the mixture of spin-singlet pairs and spin-aligned bosons occur as the chemical potential varies across the lower critical point 

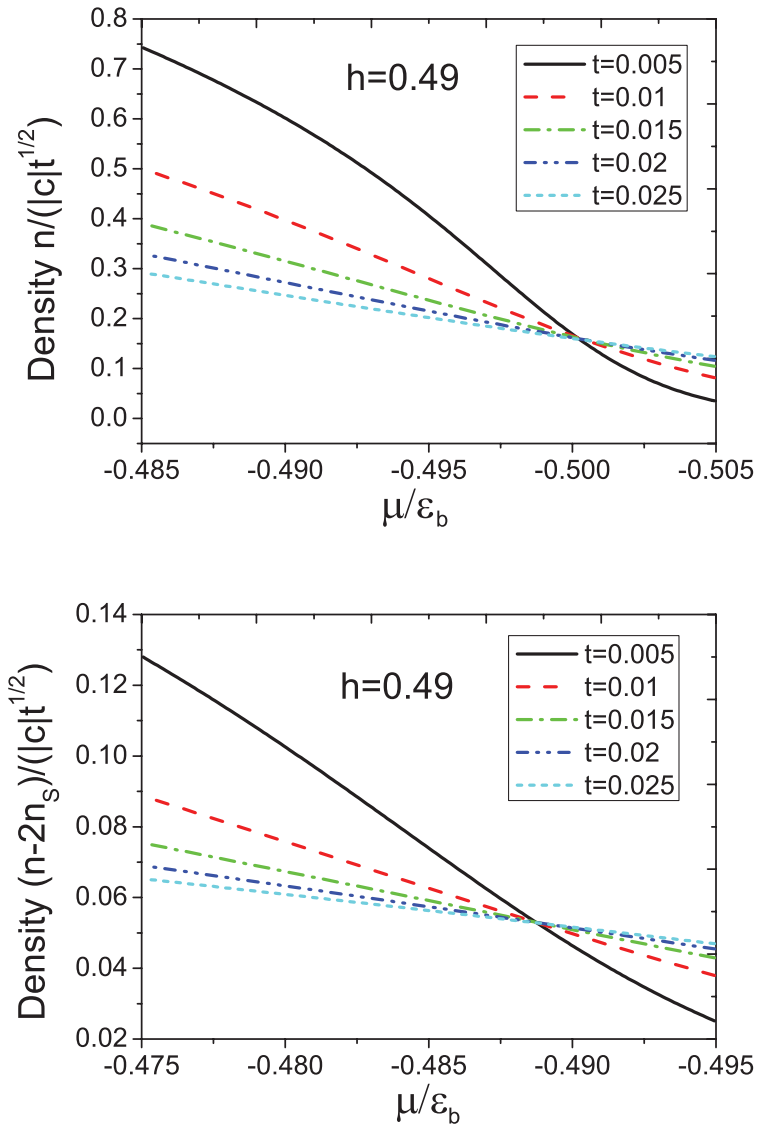

FIG. 4. (Color online) Scaled density vs chemical potential for $h=0.49$ at different temperatures $T / \epsilon_{b}=0.005,0.01,0.015,0.02$, and 0.025 . The density curves at different temperatures intersect at the critical points. This feature can be used to map out the phase boundary $V-S$ at $\tilde{\mu}_{c 2}=-0.5$ [Eq. (6)] in upper panel and the phase boundary $S-M$ at $\tilde{\mu}_{c 4} \approx-0.489$ [Eq. (12)] in the lower panel.

$\tilde{\mu}_{c 1}$ [Eq. (6)] and the upper critical point $\tilde{\mu}_{c 3}$ [Eq. (11)], respectively, see Fig. 5 . The universal scaling behavior and the zero-temperature phase diagram can be identified from the finite temperature density profiles of the trapped gas where the local chemical potentials are replaced by the harmonic trapping potential.

Furthermore, we mention that similar calculations of the scaling function can be constructed for the compressibility across all phase boundaries,

$$
\begin{aligned}
V-F: \quad \tilde{\kappa} \simeq-\frac{1}{4 \sqrt{2 \pi t}} \operatorname{Li}_{-\frac{1}{2}}\left(-e^{\frac{\tilde{\mu}-\tilde{\mu}_{c 1}}{t}}\right), \\
V-S: \quad \tilde{\kappa} \simeq-\frac{1}{\sqrt{\pi t}} \operatorname{Li}_{-\frac{1}{2}}\left(-e^{\frac{2\left(\tilde{\mu}-\tilde{\mu}_{c 2)}\right.}{t}}\right), \\
F-M: \quad \tilde{\kappa} \simeq \tilde{\kappa}_{03}-\frac{\lambda_{5}}{\sqrt{t}} \operatorname{Li}_{-\frac{1}{2}}\left(-e^{\frac{2\left(\tilde{\mu}-\tilde{\mu}_{c 3}\right)}{t}}\right), \\
S-M: \quad \tilde{\kappa} \simeq \tilde{\kappa}_{04}-\frac{\lambda_{6}}{\sqrt{t}} \operatorname{Li}_{-\frac{1}{2}}\left(-e^{\frac{\tilde{\mu}-\tilde{\mu}_{c 4}}{t}}\right) .
\end{aligned}
$$

Here $\tilde{\kappa}_{03}, \tilde{\kappa}_{04}$ are the background compressibilities in the vicinities of the critical points $\tilde{\mu}_{c 3}$ [Eq. (11)] and $\tilde{\mu}_{c 4}$ [Eq. (12)], whereas $\lambda_{5}$ and $\lambda_{6}$ are temperature-independent
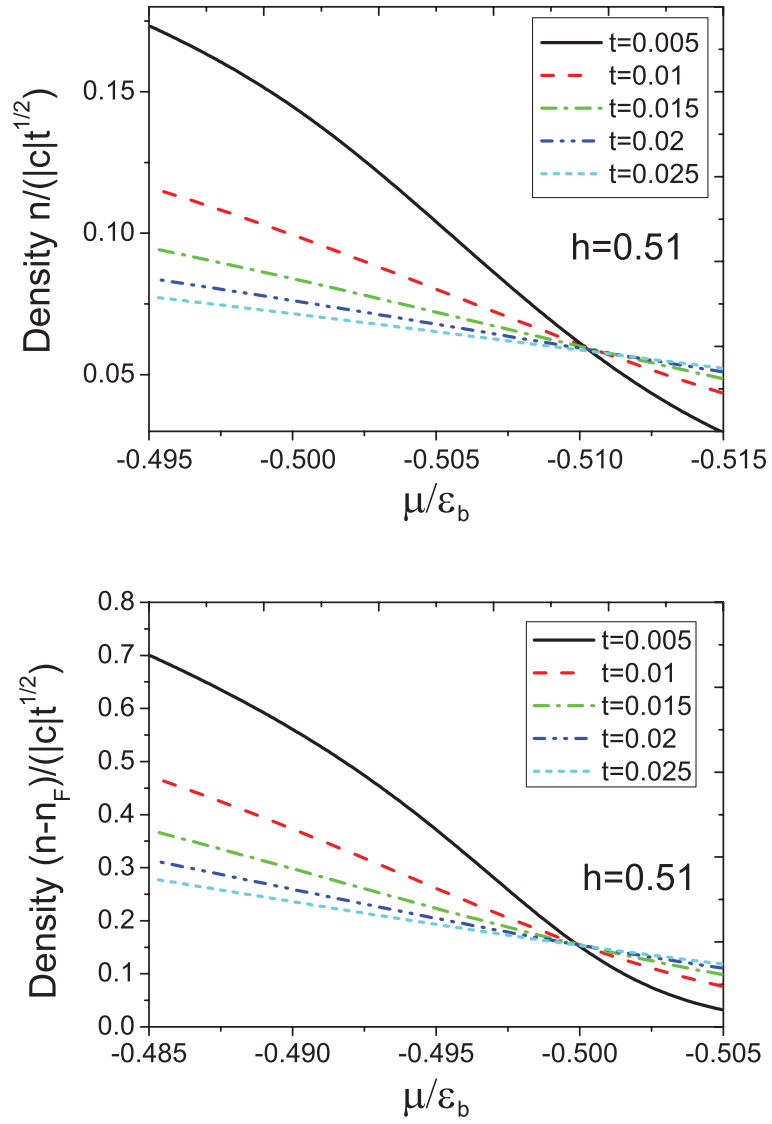

FIG. 5. (Color online) Scaled density vs chemical potential for $h=0.51$ at different temperatures $T / \epsilon_{b}=0.005,0.01,0.015,0.02$, and 0.025 . The density curves at different temperatures intersect at the critical points. This feature can be used to map out the phase boundary $V-F$ at $\tilde{\mu}_{c 1}=-h$ [Eq. (6)] in upper panel and the phase boundary $F-M$ at $\tilde{\mu}_{c 3} \approx-0.499$ [Eq. (11)] in the lower panel.

constants

$$
\begin{gathered}
\tilde{\kappa}_{03}=\frac{1}{4 \pi \sqrt{2 a}}\left(1+\frac{3 \sqrt{a}}{\pi \sqrt{2}}+\frac{3 a}{\pi^{2}}\right), \\
\tilde{\kappa}_{04}=\frac{1}{\pi \sqrt{b}}\left(1-\frac{\sqrt{b}}{2 \pi}+\frac{b}{6 \pi^{2}}\right), \\
\lambda_{5}=\frac{1}{\sqrt{\pi}}\left(1-\frac{24 \sqrt{a}}{5 \pi \sqrt{2}}+\frac{36 a}{25 \pi^{2}}\right), \\
\lambda_{6}=\frac{1}{4 \sqrt{2 \pi}}\left(1-\frac{36 \sqrt{b}}{5 \pi}+\frac{414 b}{25 \pi^{2}}\right) .
\end{gathered}
$$

Again, the critical exponents $z=2$ and $v=1 / 2$ can be read off the universal scaling function $\mathcal{F}(x)=\lambda \mathrm{Li}_{-\frac{1}{2}}(x)$ in the universal form

$$
\kappa(\mu, T)=\kappa_{0}+T^{\frac{d}{z}+1-\frac{2}{v z}} \mathcal{F}\left(\frac{\mu-\mu_{c}}{T^{\frac{1}{v z}}}\right) .
$$

\section{B. Criticality driven by a magnetic field}

The quantum phase transitions driven by a magnetic field are particularly interesting. In the phase diagram of Fig. 1, for fixed chemical potential we can vary the external field $H$ to pass the phase boundaries $S-M$ and $M-F$. At finite temperatures, 


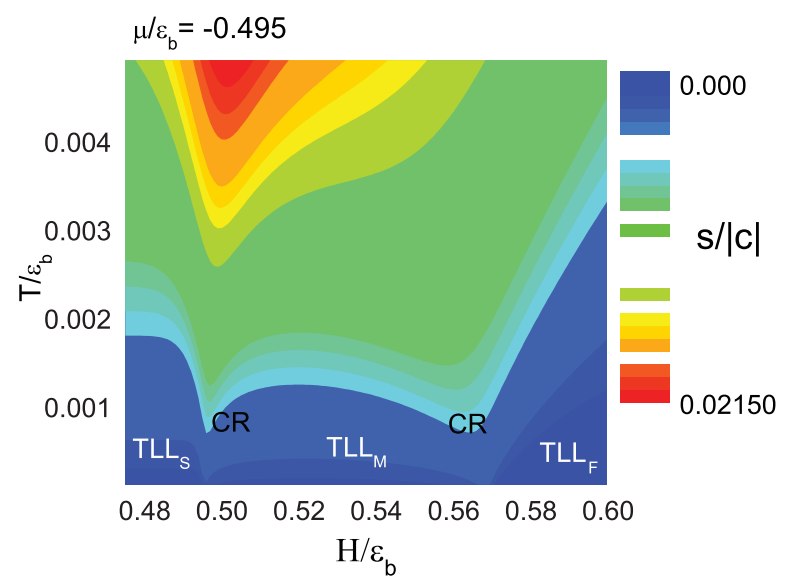

FIG. 6. (Color online) Contour plot of the entropy $S$ vs the external field $H$ for fixed chemical potential $\tilde{\mu}=-0.495$ in the $T-H$ plane. The dashed lines are determined by comparing the result from the equation of state [Eqs. (15) and (16)] and the TLL entropy (30). The crossover temperatures separate the quantum critical regimes from the TLL phases.

the three zero-temperature quantum phases, i.e., the phase of singlet pairs, ferromagnetic phase of spin-aligned atoms, and the mixed phase of pairs and single atoms, become the relativistic TLL of a bound pair $\left(\mathrm{TLL}_{S}\right)$, TLL of single atoms $\left(\mathrm{TLL}_{F}\right)$, and a two-component TLL $\left(\mathrm{TLL}_{M}\right)$ of paired and single atoms, respectively. We obtain the critical fields by converting the critical fields [Eqs. (12) and (11)]

$$
\begin{aligned}
& h_{c 1}=-\tilde{\mu}+\frac{32 \sqrt{2}}{15 \pi}\left(\tilde{\mu}+\frac{1}{2}\right)^{\frac{3}{2}}-\frac{32}{45 \pi^{2}}\left(\tilde{\mu}+\frac{1}{2}\right)^{2}, \\
& h_{c 2}=-\tilde{\mu}+\frac{1}{2}\left(\frac{15 \pi}{4}\right)^{\frac{2}{3}}\left(\tilde{\mu}+\frac{1}{2}\right)^{\frac{2}{3}}-\frac{5}{8}\left(\tilde{\mu}+\frac{1}{2}\right) .
\end{aligned}
$$

These critical fields and the crossover temperatures can be observed in the contour plot of the entropy in the $T-H$ plane, see Fig. 6. The low-energy TLL physics breaks down at the crossover temperature (dashed lines) where the dispersion of either bound pairs or unpaired single atoms becomes nonrelativistic. In particular, in the vicinity of the quantum critical points $h_{c 1}$ and $h_{c 2}$, the system exhibits two different quantum critical regimes. From Eqs. (34), (35), and (37), we find the scaling function in the critical regime near the critical point (50)

$$
S-M: \quad\left\{\begin{array}{l}
\tilde{n} \simeq \tilde{n}_{05}-\lambda_{7} \sqrt{t} \operatorname{Li}_{\frac{1}{2}}\left(-e^{\frac{h-h_{c 1}}{t}}\right), \\
\tilde{M} \simeq-\lambda_{8} \sqrt{t} \operatorname{Li}_{\frac{1}{2}}\left(-e^{\frac{h-h_{c 1}}{t}}\right) \\
\tilde{\kappa} \simeq \tilde{\kappa}_{05}-\frac{\lambda_{9}}{\sqrt{t}} \mathrm{Li}_{-\frac{1}{2}}\left(-e^{\frac{h-h_{c 1}}{t}}\right)
\end{array}\right.
$$

where the constants are given by

$$
\begin{aligned}
\tilde{n}_{05} & =\frac{\sqrt{d}}{\pi}\left(1-\frac{\sqrt{d}}{6 \pi}+\frac{d}{36 \pi^{2}}\right), \\
\tilde{\kappa}_{05} & =\frac{1}{\pi \sqrt{d}}\left(1-\frac{\sqrt{d}}{2 \pi}+\frac{d}{6 \pi^{2}}\right), \\
\lambda_{7} & =\frac{1}{4 \sqrt{2 \pi}}\left(1-\frac{32 \sqrt{d}}{5 \pi}+\frac{848 d}{75 \pi^{2}}\right),
\end{aligned}
$$

$$
\begin{aligned}
& \lambda_{8}=\frac{1}{4 \sqrt{2 \pi}}\left(1-\frac{16 \sqrt{d}}{5 \pi}+\frac{8 d}{15 \pi^{2}}\right), \\
& \lambda_{9}=\frac{1}{4 \sqrt{2 \pi}}\left(1-\frac{36 \sqrt{d}}{5 \pi}+\frac{414 d}{25 \pi^{2}}\right),
\end{aligned}
$$

with

$$
\begin{aligned}
& d=2\left(\tilde{\mu}+\frac{1}{2}\right)\left(1-\frac{\sqrt{2}}{9 \pi} \sqrt{\tilde{\mu}+\frac{1}{2}}\right) \\
& e=\frac{1}{2}\left(\frac{15 \pi}{4}\right)^{\frac{2}{3}}\left(\tilde{\mu}+\frac{1}{2}\right)^{\frac{2}{3}} .
\end{aligned}
$$

In the vicinity of the quantum critical points $h_{c 2}$, we obtain the scaling forms

$$
F-M:\left\{\begin{array}{l}
\tilde{n} \simeq \tilde{n}_{06}-\lambda_{10} \sqrt{t} \operatorname{Li}_{\frac{1}{2}}\left(-e^{\frac{\alpha\left(h-h_{c 2}\right)}{t}}\right), \\
\tilde{M} \simeq \tilde{M}_{06}+\lambda_{11} \sqrt{t} \operatorname{Li}_{\frac{1}{2}}\left(-e^{\frac{\alpha\left(h-h_{c 2}\right)}{t}}\right), \\
\tilde{\kappa} \simeq \tilde{\kappa}_{06}-\frac{\lambda_{12}}{\sqrt{t}} \operatorname{Li}_{-\frac{1}{2}}\left(-e^{\frac{\alpha\left(h-h_{c 2}\right)}{t}}\right),
\end{array}\right.
$$

where

$$
\begin{aligned}
& \tilde{n}_{06}=\frac{\sqrt{e}}{2 \pi \sqrt{2}}\left(1+\frac{\sqrt{e}}{\pi \sqrt{2}}+\frac{e}{2 \pi^{2}}\right), \\
& \tilde{\kappa}_{06}=\frac{1}{4 \pi \sqrt{2 e}}\left(1+\frac{3 \sqrt{e}}{\pi \sqrt{2}}+\frac{3 e}{\pi^{2}}\right) . \\
& \lambda_{10}=\frac{1}{2 \sqrt{\pi}}\left(1-\frac{16 \sqrt{e}}{5 \pi \sqrt{2}}-\frac{8 e}{25 \pi^{2}}\right), \\
& \lambda_{11}=\frac{4 \sqrt{e}}{5 \sqrt{2} \pi^{3 / 2}}\left(1-\frac{3 \sqrt{e}}{5 \pi \sqrt{2}}\right) \\
& \lambda_{12}=\frac{1}{\sqrt{\pi}}\left(1-\frac{24 \sqrt{e}}{5 \pi \sqrt{2}}+\frac{36 e}{25 \pi^{2}}\right),
\end{aligned}
$$

with

$$
\alpha=-\frac{8}{5 \pi}\left(\frac{15 \pi}{4}\right)^{\frac{1}{3}}\left(\tilde{\mu}+\frac{1}{2}\right)^{\frac{1}{3}} .
$$

In this case, the background density $\tilde{M}_{06}=\tilde{n}_{06}$ is equal to the total density at the critical point. The density (or magnetization) and compressibility can be recast into the universal scaling form

$$
\begin{aligned}
& M(h, T)=n_{0}+T^{\frac{d}{z}+1-\frac{1}{v z}} \mathcal{G}\left(\frac{\alpha\left(h-h_{c}\right)}{T^{\frac{1}{\nu z}}}\right), \\
& \kappa(\mu, T)=\kappa_{0}+T^{\frac{d}{z}+1-\frac{2}{v z}} \mathcal{F}\left(\frac{\alpha\left(h-h_{c}\right)}{T^{\frac{1}{\nu z}}}\right),
\end{aligned}
$$

with the same critical exponents as those for quantum criticality driven by the chemical potential, i.e., the dynamical critical exponent $z=2$ and the correlation length exponent $v=1 / 2$.

In Fig. 7 we show the magnetization as a function of the external field for different temperatures for a fixed chemical potential. All curves intersect at the critical point $h_{c 1}$ without background magnetization. However, these curves intersect at the upper critical point $h_{c 2}$ with appropriate subtraction of the background magnetization. We can obtain 

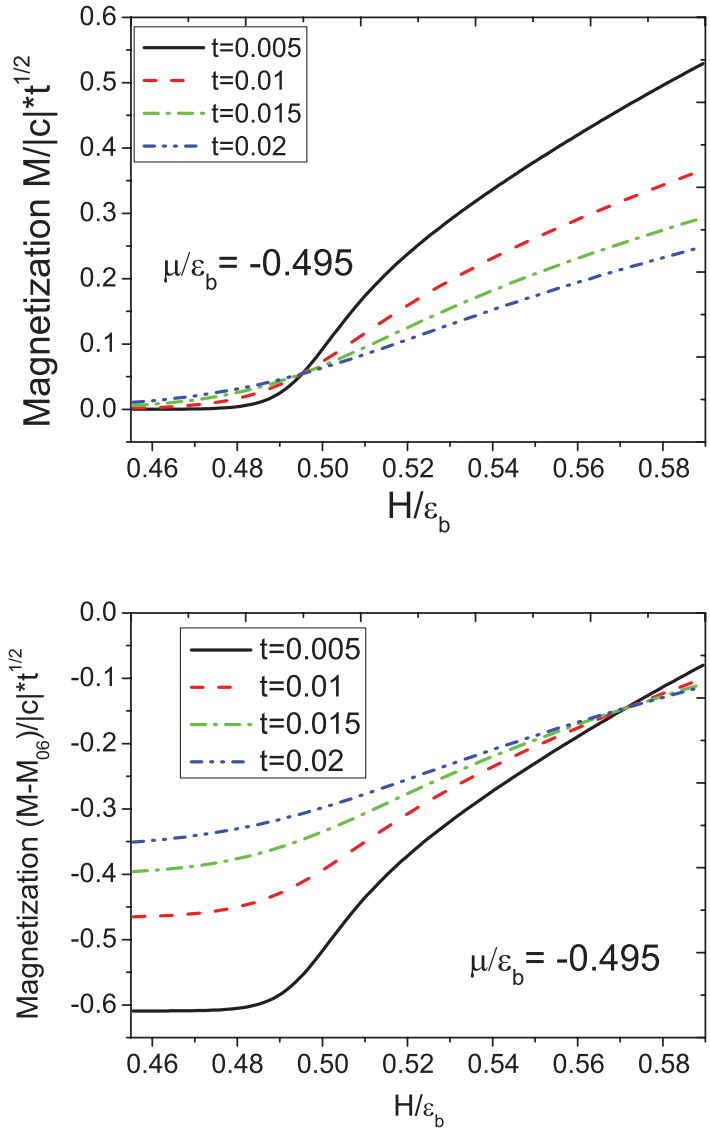

FIG. 7. (Color online) Magnetization vs external field for $\tilde{\mu}=$ -0.495 . Upper panel: the intersection point of the magnetization curves at different temperatures gives the critical external field $h_{c 1}$ at the boundary $S-M$. Lower panel: after a proper subtraction of the background magnetization $\tilde{M}_{06}=n(t)$, the intersection point of the magnetization curves at different temperatures gives the critical external field $h_{c 2}$ at the boundary $F-M$.

similar scaling behavior for the densities near the critical points $h_{c 1}$ and $h_{c 2}$ like that presented in Figs. 4 and 5. It turns out that magnetization can be used to map out the bulk phase diagram through the $1 \mathrm{D}$ trapped gas at finite temperatures.

\section{CONCLUSION}

Using the TBA equations, we have studied the quantum phase diagram, thermodynamics, and quantum critical behavior of one-dimensional spin-1 bosons with strongly repulsive density-density and antiferromagnetic spin-exchange interactions. We have determined with high precision the equation of state from which the TLL thermodynamics, universal scaling functions, and critical exponents have been obtained. The universality class of quantum criticality has also been discussed.

The scaling forms of the density, compressibility, magnetization, and susceptibility associated with the phase transitions driven by the chemical potential and magnetic field were rescaled to the universality class of quantum criticality of free fermions with critical exponent $z=2$ and correlation length exponent $v=1 / 2$. It thus turns out that the quantum criticality of the spin-1 Bose gas belongs to the same universality class as spin-1/2 attractive fermions [38] due to the hard-core nature of the two coupled Tonks-Girardeau gases. We have also shown that the quantum criticality in 1D systems involves a universal crossover from a TLL with linear dispersion to free fermions with a quadratic dispersion near the critical point. These scaling forms for the thermodynamic properties across the phase boundaries illustrate the physical origin of quantum criticality in this system, where the singular part of the thermodynamic properties involves a sudden change of density of state for either pairs or unpaired single atoms. The phase diagram, the TLL thermodynamics, and critical properties of the bulk system can be mapped out from the density and magnetization profiles of the trapped spinor gas at finite temperatures. Our results open the way to further study of such universal features of 1D many-body physics in experiments with ultracold atoms.

\section{ACKNOWLEDGMENTS}

This work has been partially supported by the Australia Research Council. C.C.N.K. thanks CAPES (Coordenação de Aperfeicoamento de Pessoal de Nivel Superior) for financial support. He also thanks the Department of Theoretical Physics for their hospitality. A.F. thanks CNPq (Conselho Nacional de Desenvolvimento Cientifico e Tecnológico) for financial support.
[1] T.-L. Ho, Phys. Rev. Lett. 81, 742 (1998); T.-L. Ho and S. K. Yip, ibid. 84, 4031 (2000).

[2] T. Ohmi and K. Machida, J. Phys. Soc. Jpn. 67, 1822 (1988).

[3] D. M. Stamper-Kurn, M. R. Andrews, A. P. Chikkatur, S. Inouye, H.-J. Miesner, J. Stenger, and W. Ketterle, Phys. Rev. Lett. 80, 2027 (1998).

[4] H.-J. Miesner, D. M. Stamper-Kurn, J. Stenger, S. Inouye, A. P. Chikkatur, and W. Ketterle, Phys. Rev. Lett. 82, 2228 (1999).

[5] M. R. Matthews, B. P. Anderson, P. C. Haljan, D. S. Hall, C. E. Wieman, and E. A. Cornell, Phys. Rev. Lett. 83, 2498 (1999).

[6] M. D. Barrett, J. A. Sauer, and M. S. Chapman, Phys. Rev. Lett. 87, 01040 (2001).
[7] M.-S. Chang, Q. Qin, W. Zhang, L. You, and M. S. Chapman, Nat. Phys. 1, 111 (2005).

[8] H. Moritz, T. Stöferle, M. Köhl, and T. Eslinger, Phys. Rev. Lett. 91, 250402 (2003).

[9] B. Paredes, A. Widera, V. Murg, O. Mandel, S. Folling, I. Cirac, G. V. Shlyapnikov, T. W. Hansch, and I. Bloch, Nature 429, 277 (2004).

[10] T. Kinoshita, T. Wenger, and D. S. Weiss, Science 305, 1125 (2004).

[11] T. Kinoshita, T. Wenger, and D. S. Weiss, Phys. Rev. Lett. 95, 190406 (2005).

[12] T. Kinoshita et al., Nature 440, 900 (2006). 
[13] A. H. van Amerongen et al., Phys. Rev. Lett. 100, 090402 (2008).

[14] T. Kitagawa et al., Phys. Rev. Lett. 104, 255302 (2010).

[15] J. Armijo, T. Jacqmin, K. V. Kheruntsyan, and I. Bouchoule, Phys. Rev. Lett. 105, 230402 (2010); J. Armijo, T. Jacqmin, K. Kheruntsyan, and I. Bouchoule, Phys. Rev. A 83, 021605(R) (2011).

[16] E. Haller et al., Science 325, 1224 (2009).

[17] Y. Liao et al., Nature 467, 567 (2010).

[18] S. Giorgini, L. P. Pitaevskii, and S. Stringari, Rev. Mod. Phys. 80, 1215 (2008).

[19] M. A. Cazalilla, R. Citro, T. Giamarchi, E. Orignac, and M. Rigol, Rev. Mod. Phys. 83, 1405 (2011).

[20] E. Eisenberg and E. H. Lieb, Phys. Rev. Lett. 89, 220403 (2002).

[21] K. Yang and Y.-Q. Li, Int. J. Mod. Phys. B 17, 1027 (2003).

[22] C. N. Yang, Phys. Rev. Lett. 19, 1312 (1967); M. Gaudin, Phys. Lett. A 24, 55 (1967).

[23] B. Sutherland, Phys. Rev. Lett. 20, 98 (1968).

[24] Y.-Q. Li, S.-J. Gu, Z.-J. Ying, and U. Eckern, Europhys. Lett. 61, 368 (2003).

[25] X.-W. Guan, M. T. Batchelor, and M. Takahashi, Phys. Rev. A 76, 043617 (2007).

[26] J. Cao, Y. Jiang, and Y. Wang, Europhys. Lett. 79, 30005 (2007).

[27] Y. Jiang, J. Cao, and Y. Wang, J. Phys. A 44, 345001 (2011).

[28] F. H. L. Essler, G. V. Shlyapnikov, and A. M. Tsvelik, J. Stat. Mech. (2009) P02027.

[29] J. Y. Lee, X.-W. Guan, M. T. Batchelor, and C. Lee, Phys. Rev. A 80, 063625 (2009).

[30] G. V. Shlyapnikov and A. M. Tsvelik, New J. Phys. 13, 065012 (2011).
[31] S. Sachdev, Quantum Phase Transitions (Cambridge University, Cambridge, England, 1999).

[32] M. P. A. Fisher, P. B. Weichman, G. Grinstein, and D. S. Fisher, Phys. Rev. B 40, 546 (1989).

[33] N. Gemelke, X. Z. Zhang, C.-L. Hung, and C. Chin, Nature 460, 995 (2009); C.-L. Hung, X. Z. Zhang, N. Gemelke, and C. Chin, ibid. 470, 236 (2011).

[34] P. Cladé, C. Ryu, A. Ramanathan, K. Helmerson, and W. D. Phillips, Phys. Rev. Lett. 102, 170401 (2009); N. Gemelke, X. Zhang, C.-L. Hung, and C. Chin, Nature 460, 995 (2009).

[35] M. Campostrini and E. Vicari, Phys. Rev. Lett. 102, 240601 (2009); 103, 269901(E) (2009).

[36] Q. Zhou and T.-L. Ho, Phys. Rev. Lett. 105, 245702 (2010).

[37] K. R. A. Hazzard and E. J. Mueller, Phys. Rev. A 84, 013604 (2011).

[38] X.-W. Guan and T.-L. Ho, Phys. Rev. A 84, 023616 (2011).

[39] X.-W. Guan and M. T. Batchelor, J. Phys. A 44, 102001 (2011).

[40] X. Yin, X.-W. Guan, Y. Zhang, and S. Chen, Phys. Rev. A 85, 013608 (2012).

[41] C. N. Yang and C. P. Yang, J. Math. Phys. 10, 1115 (1969).

[42] N. Navon, S. Nascimbene, F. Chevy, and C. Salomon, Science 328, 729 (2010).

[43] M. Horikoshi, S. Nakajima, M. Ueda, and T. Mukaiyama, Science 327, 442 (2010).

[44] T.-L. Ho and Q. Zhou, Nat. Phys. 6, 131 (2009).

[45] H. W. J. Blöte, J. L. Cardy, and M. P. Nightingale, Phys. Rev. Lett. 56, 742 (1986); I. Affleck, ibid. 56, 746 (1986).

[46] Y. Maeda, C. Hotta, and M. Oshikawa, Phys. Rev. Lett. 99, 057205 (2007).

[47] E. Zhao, X.-W. Guan, W. V. Liu, M. T. Batchelor, and M. Oshikawa, Phys. Rev. Lett. 103, 140404 (2009). 